\title{
MINHOCAS EXÓTICAS COMO INDICADORAS DE PERTURBAÇÃO ANTRÓPICA EM ÁREAS DE FLORESTA ATLÂNTICA
}

\author{
Juliana O. Fernandes ${ }^{1}$, Marcio Uehara-Prado ${ }^{1}$ \& George G. Brown ${ }^{2}$ \\ ${ }^{1}$ Museu de História Natural e Departamento de Zoologia, Instituto de Biologia, Universidade Estadual \\ de Campinas, CP 6109 CEP13084-971 Campinas-SP, Brasil. E-mail: juliana_olifer@yahoo.com.br, \\ muprado@yahoo.com \\ ${ }^{2}$ Embrapa Florestas, CP 319, CEP 83411-000 Colombo-PR, Brasil. E-mail: browng@enpf.embrapa.br
}

Fernandes, J. O., M. Uehara-Prado \& G. G. Brown. 2010. Minhocas exóticas como indicadoras de perturbação antrópica em áreas de floresta atlântica. Acta Zoológica Mexicana (n.s.), Número Especial 2: 211-217.

RESUMO. No presente trabalho verificou-se o efeito de perturbação antrópica na incidência de minhocas exóticas e nativas ativas na superfície do solo de uma área de Floresta Atlântica do Estado de São Paulo, Brasil. Os oligoquetos foram amostrados usando armadilhas de queda (pitfall) em duas áreas, uma onde houve corte raso e queima, e outra onde houve corte seletivo de espécies lenhosas. A maior parte dos indivíduos $(91,4 \%)$ foi amostrada na área onde houve corte raso e queima, e todos eram espécies exóticas (Amynthas sp., A. gracilis, A. corticis) ou peregrinas (Pontoscolex corethrurus). O contraste no número de indivíduos entre as duas áreas sugere que a perturbação antrópica influencia a atividade superficial e a abundância das espécies exóticas de minhocas e que estas apresentam potencial de uso como indicadores biológicos.

Palavras chave: Invertebrados, bioindicador, espécies exóticas invasoras, Oligochaeta.

Fernandes, J. O., M. Uehara-Prado \& G. G. Brown. 2010. Lombrices de tierra exóticas como indicadoras de perturbación antrópica en áreas de mata atlántica. Acta Zoológica Mexicana (n.s.), Número Especial 2: 211-217.

RESUMEN. En el presente trabajo se evaluó el efecto de la perturbación antrópica en la incidencia de lombrices exóticas y nativas activas en la superficie del suelo en un área de bosque atlántico del estado de São Paulo, Brasil. Los oligoquetos fueron muestreados usando trampas (pitfall) en dos áreas, una en donde hubo corte raso y quema y otra en donde solamente hubo corte selectivo. La mayor parte de los individuos (91.4\%) fue colectada en el área en donde hubo corte raso y quema y todos eran especies exóticas (Amynthas sp., A. gracilis, A. corticis) o peregrinas (Pontoscolex corethrurus). El contraste del número de individuos entre las dos áreas sugiere que la perturbación antrópica influye en la actividad superficial y la abundancia de especies exóticas de lombrices y que éstas presentan un uso potencial como indicadores biológicos.

Palabras clave: Invertebrados, bioindicador, especies exóticas invasoras, Oligochaeta.

Recibido: 16/05/2008; aceptado: 08/01/2010. 


\section{INTRODUÇÃO}

O resultado da atividade antrópica nos ambientes naturais pode ser medido e interpretado por mudanças na abundância, diversidade e composição de grupos indicadores que dependem de certos recursos do ecossistema (Brown 1997). Muitos pesquisadores têm utilizado vertebrados e plantas superiores como grupos indicadores de perturbação, no entanto os invertebrados têm se mostrado mais sensíveis a modificações no habitat e à intensidade de impacto (Lewinsohn et al. 2005).

A presença da população de minhocas em um sistema depende das condições climáticas (precipitação, temperatura, e umidade do ar), edáficas ( $\mathrm{pH}$, matéria orgânica, nutrientes, tipo, cor, umidade e temperatura), vegetais (tipo de vegetação) e topográficas (declividade e posição fisiografica) da área (Reynold \& Jordan 1975). As espécies exóticas e peregrinas, por serem mais adaptadas a variações climáticas e mudanças edáficas, se beneficiam quando há conversão de florestas e pastagens nativas para áreas de cultivos, havendo aumento na densidade populacional destas minhocas (Fragoso et al. 1999).

As espécies exóticas pertencentes à família Megascolecidae (por exemplo, do gênero Amynthas Kinberg) e a peregrina Pontoscolex corethrurus (Müller 1857) possuem forte afinidade por ambientes antrópicos, sendo facilmente encontradas em solos urbanos, áreas agrícolas e florestas secundárias (Righi 1999, Brown et al. 2006). Os trabalhos que tratam as minhocas como bioindicadores estão relacionados principalmente aos agroecossistemas (de contaminação por agrotóxicos, práticas agrícolas, e manejo e fertilidade do solo) (Peixoto \& Marochi 1996, Langenbach et al. 2002, Brown et al. 2003, Santos et al. 2003, Nunes et al. 2007, Römbke et al. 2007), havendo poucos estudos de como suas populações reagem a mudanças em ambientes naturais como as Florestas Tropicais.

Desta forma, o objetivo deste estudo foi quantificar a incidência de minhocas exóticas ativas na superfície do solo de uma área de Floresta Atlântica, com diferentes graus de alteração antrópica.

\section{MATERIAL E MÉTODOS}

O estudo foi conduzido no município de São Luiz do Paraitinga, São Paulo (23ํำ'S, $45^{\circ} 20^{\prime} \mathrm{W}$ ), próximo às escarpas da Serra do Mar, no planalto de Paraitinga-Paraibuna. As altitudes na região variam de 870 a $1.100 \mathrm{~m}$. O clima é úmido sem estação seca, a temperatura média anual é de $20^{\circ} \mathrm{C}\left(\min .12^{\circ} \mathrm{C}\right.$, máx. $\left.27^{\circ} \mathrm{C}\right)$ e a precipitação média é de $2.180 \mathrm{~mm} /$ ano. Predominam na região solos do tipo Latossolo Vermelho-Amarelo, Cambissolo e solos Litólicos (RADAMBRASIL 1983). A vegetação original foi classificada por Veloso et al. (1991) como Floresta Ombrófila Densa.

O Núcleo Santa Virgínia, criado em 1989, possui área de 4.894 ha, está localizado na porção sudeste do município de São Luiz do Paraitinga, no Parque Estadual da 
Serra do Mar $\left(23^{\circ} 17^{\prime}-23^{\circ} 24^{\prime} \mathrm{S}, 4^{\circ} 03^{\prime}-45^{\circ} 11^{\prime} \mathrm{W}\right)$ e inserido em um grande contínuo de Floresta Atlântica. A região do parque é considerada "área de extrema importância biológica" para a fauna e a flora, sendo, portanto, da mais alta prioridade para conservação da Floresta Atlântica, segundo critérios estabelecidos pelo Ministério do Meio Ambiente (MMA 2000).

Na década de 1960, ocorreu em parte da Floresta Atlântica no Núcleo Santa Virgínia, corte raso e queimadas, que geraram a substituição da floresta por pastagens (Melinis menutiflora - Gramineae) para pecuária e plantação de Eucalyptus spp. para produção de carvão. Atualmente, essa área se apresenta na forma de mosaico, composto por áreas de floresta madura, pastagens, plantios de Eucalyptus spp. e florestas secundárias em diferentes estágios de regeneração (Tabarelli \& Mantovani 1999). As famílias vegetais Melastomataceae, Rubiaceae, Lauraceae, e Myrtaceae estão presentes em toda área do Núcleo; contudo, quanto mais recente a regeneração da floresta, maior é a presença das famílias Melastomataceae e Rubiaceae e, quanto mais próxima a floresta do clímax, maior é a importância das famílias Myrtaceae e Lauraceae (Tabarelli \& Mantovani 1999).

Para avaliar a resposta das minhocas à perturbação antrópica, foram comparadas duas áreas, uma onde houve o corte raso, queimada e a substituição da mata por pastagem (Trilha do Poço do Pito), e outra onde houve corte seletivo de espécies lenhosas (Trilha do Ipiranga, acima da Base Itamambuca). Atualmente, a área amostrada na trilha do Ipiranga está coberta na sua maior parte por floresta madura, enquanto a outra área está coberta por vegetação com idades entre 17 e 40 anos de regeneração (Comunicação pessoal de João Paulo Villani, Tabarelli \& Mantovani 1999).

Em cada área foram instaladas seis unidades amostrais, que distavam pelo menos $100 \mathrm{~m}$ entre si. Cada unidade amostral era constituída por cinco armadilhas do tipo pitfall (copos plástico transparentes de $500 \mathrm{ml}$, com $9 \mathrm{~cm}$ de diâmetro e $12 \mathrm{~cm}$ de altura) nivelados ao solo, com a abertura protegida por uma cobertura de isopor dispostas linearmente, distantes dois metros entre si e contendo como líquido conservante propileno glicol $30 \%$ e formol $0,1 \%$. As 30 armadilhas em cada área ficaram abertas seis dias por mês, de novembro de 2004 a maio de 2005 .

As minhocas coletadas foram fixadas em formol $2 \%$ e conservadas em álcool $70 \%$. No gênero Amynthas, apenas as minhocas com clitelo (adultas) puderam ser identificadas até espécie. Os exemplares coletados foram identificados pelo terceiro autor e depositados no Museu de História Natural da Universidade Estadual de Campinas.

\section{RESULTADOS}

Após 60.480 horas de amostragem, foi capturado um total de 58 indivíduos representando quatro espécies de duas famílias da Subordem Lumbricina: Amynthas gracilis 
(Kinberg 1867) e Amynthas corticis (Kinberg 1867), ambas espécies exóticas da família Megascolecidae, e Pontoscolex corethrurus (Müller 1857) e Glossoscolex sp. (juvenil), espécies da família Glossoscolecidae. Até o momento, nenhuma minhoca era conhecida da reserva, sendo esse o primeiro registro de espécies do Núcleo Santa Virgínia.

A maior parte dos indivíduos encontrados $(91,4 \%)$ foi coletada onde houve corte raso e queima; nessa área todos eram espécies exóticas ou peregrinas (Tabela I). As espécies de Amynthas predominaram, representando 95\% de todas as minhocas coletadas, sendo mais abundantes na área mais perturbada (Poço do Pito). $\mathrm{Na}$ área menos perturbada, apenas cinco indivíduos foram encontrados; destes, dois eram do gênero Amynthas (exóticas) e os demais eram espécies peregrinas ou nativas (Tabela I).

\section{DISCUSSÃO}

No presente estudo foram recuperados apenas dois indivíduos de $P$. corethrurus e um de Glossoscolex sp. (não pigmentada). Estas espécies endógeas sobem pouco à superfície do solo e, portanto, são pouco coletadas usando o método de pitfall. Várias espécies do gênero Glossoscolex habitam a Floresta Atlântica no Estado de São Paulo (Brown \& James 2006), e a espécie P. corethrurus é a minhoca com a mais ampla distribuição do país, sendo encontrada em quase todos os estados, principalmente em ecossistemas perturbados, incluindo agroecossistemas e ecossistemas naturais (Brown et al. 2006).

A grande maioria dos indivíduos coletados pertencia à família Megascolecidae, gênero Amynthas, minhocas amplamente conhecidas e distribuídas no Brasil e com

Tabela I. Número de indivíduos e espécies de minhocas exóticas e nativas coletadas por armadilha de queda (pitfall), no Núcleo Santa Virgínia, em áreas com histórico de corte raso e queima (maior perturbação), ou corte seletivo de espécies lenhosas (menor perturbação).

\begin{tabular}{lllcc}
\hline \multicolumn{1}{c}{ Família } & \multicolumn{1}{c}{ Espécies } & Origem & Corte raso e queima & Corte seletivo \\
\hline Megascolecidae $^{1}$ & Amynthas corticis & Exótica & 19 & 2 \\
& Amynthas gracilis & Exótica & 9 & 0 \\
& Amynthas sp. (juvenis) & Exóticas & 23 & 2 \\
Glossoscolecidae & Pontoscolex corethrurus & Peregrina & 2 & 0 \\
& Glossoscolex sp. & Nativa & 0 & 1 \\
Total & - & - & 53 & 5 \\
Espécies exóticas (\%) & - & - & 96 & 80 \\
Exóticas+peregrinas (\%) & - & - & 100 & 80 \\
\hline
\end{tabular}

${ }^{1}$ Família que no Brasil possui apenas espécies introduzidas (Righi 1999, Brown \& James 2006).

${ }^{2}$ Espécie peregrina, originária do Platô das Guianas (Righi 1984). 
alto poder de invasão a ambientes antrópicos (Brown et al. 2006). São fáceis de encontrar e abundantes em ambientes urbanos, e são conhecidas popularmente como minhoca "louca", "brava", "saltadora" ou "puladeira". O comportamento ativo na superfície do solo, a locomoção, o modo reprodutivo (partenogênese) e o ciclo de vida curto são as principais razões de seu sucesso como colonizadoras (Brown et al. 2006).

A densidade de minhocas exóticas coletadas, especialmente do gênero Amynthas, foi diferente entre as duas áreas avaliadas no presente estudo, sendo maior na área onde houve corte raso e queima, e menor na área onde houve apenas corte seletivo de espécies, sugerindo que a perturbação antrópica pode favorecer a invasão e o estabelecimento dessas espécies (Hendrix 2006). Porém, no presente estudo é impossível saber quando e porque as minhocas exóticas chegaram à reserva, devido à ausência de avaliações prévias das espécies do local. Contudo, sabe-se que as espécies exóticas têm sido transportadas pela ação humana a muitas áreas perturbadas e com vegetação nativa (Brown et al. 2006, Hendrix 2006), onde elas podem chegar a se estabelecer e se espalhar, dependendo das condições locais. Nesse sentido, apesar do método de coleta de minhocas usado no presente estudo (pitfall) não ser ideal para quantificar populações de minhocas no campo, ele pode ser de utilidade para indicar a atividade e abundância de espécies que se movem na superfície do solo (Baker \& Lee 1993, Hendrix 2002, Callaham et al. 2003). Dentre essas, estão as minhocas exóticas do gênero Amynthas, que não são bem coletadas usando o método de catação (triagem) manual do solo, devido aos seus rápidos movimentos de fuga quando perturbadas (Baretta et al. 2007).

Portanto, o maior número de indivíduos do gênero Amynthas coletados nas armadilhas da área perturbada indica maior abundância desses animais nessa área e/ou condições mais adequadas para atividade na superfície do solo desse local. A maior abundância e proporção de espécies exóticas e peregrinas na área perturbada podem também indicar maior preferência dessas minhocas a esse habitat ou a maior facilidade de sua colonização. Contudo, para realizar uma avaliação mais precisa da abundância das minhocas nativas e exóticas do local, é necessário usar os métodos tradicionais de coleta de minhocas (triagem manual e/ou extração com formol diluído para coletar as espécies mais velozes), já que as armadilhas não proporcionam uma medida direta da abundância específica de minhocas no local, especialmente das espécies endógeas que pouco se locomovem na superfície do solo (Baker \& Lee 1993).

Estudos sobre a dinâmica populacional, ecologia, biologia e diversidade das espécies de minhocas exóticas invasoras e suas relações com os ambientes em que vivem são necessários para evitar sua invasão em ecossistemas naturais e/ou controlar essas populações em locais já invadidos (Brown et al. 2006). Esses estudos devem, preferivelmente, contemplar o uso de diversos métodos de coleta (Baretta et al. 2007) e avaliação da atividade e poder de invasão das minhocas exóticas, especialmente do 
gênero Amynthas. Nesse sentido, o uso das armadilhas pode ser útil para realizar uma avaliação da atividade de minhocas na superfície e do potencial de invasão/colonização das mesmas, algo mais difícil de realizar usando os métodos tradicionais de coleta de minhocas (Callaham et al. 2003).

No presente trabalho, as espécies do gênero Amynthas mostraram um potencial para bioindicação de ambientes perturbados em áreas de Floresta Atlântica, e também de seu potencial para colonizar novos ambientes, considerando sua locomoção freqüente na superfície do solo. Estudos mais extensos e detalhados contribuirão para um melhor aproveitamento dessas espécies como indicadoras, e do potencial real do uso de armadilhas de queda (pitfall) em programas de avaliação e monitoramento ambiental.

\section{AGRADECIMENTOS}

Os autores agradecem: João Paulo Villani e os funcionários do Núcleo Sta. Virgínia pelo apoio e camaradagem durante o período de execução do trabalho; Artur N. Furegatti e Keith S. Brown Jr. pelo apoio logístico antes, durante e depois das amostragens; os estagiários do projeto "Artrópodes como indicadores biológicos de perturbação antrópica em Floresta Atlântica" pelo auxílio na triagem do material; todos os que colaboraram na instalação dos pitfalls; André V. L. Freitas pelas sugestões pertinentes no delineamento experimental e na versão final do texto; Inara R. Leal pelas sugestões pertinentes na versão final do texto; o Instituto Florestal do Estado de São Paulo pela autorização de pesquisa. M. Uehara-Prado e G.G. Brown agradecem ao Conselho Nacional de Desenvolvimento Científico e Tecnológico pela bolsa de doutoramento e de pesquisa, respectivamente.

\section{LITERATURA CITADA}

Baker, G. H. \& K. E. Lee. 1993. Earthworms. Pp. 359-371. In: M. R. Carter (Ed.). Soil sampling and methods of analysis. Lewis Publishers, Boca Raton.

Baretta, D., G. G. Brown, S. W. James \& E. J. B. N. Cardoso. 2007. Earthworm populations sampled using collection methods in Atlantic forests with Araucaria angustifolia. Scientia Agricola. 64(4): 484-392.

Brown Jr., K. S. 1997. Diversity, disturbance and sustainable use of Neotropical forests: insects for conservation monitoring. Journal of Insect Conservation. 1: 1-8.

Brown, G. G., N. P. Benito, A. Pasini, K. D. Sautter, M. de F. Guimarães \& E. Torres. 2003. Notillage greatly increases earthworm populations in Paraná state, Brazil. Pedobiologia. 47: 764-771.

Brown, G. G. \& S. W. James. 2006. Earthworms biodiversity in São Paulo state, Brazil. European Journal of Soil Science. 42: 145-149.

Brown, G. G., S. W. James, A. Pasini, D. H. Nunes, N. P. Benito, P. T. Martins \& K. D. Sautter. 2006. Exotic, peregrine, and invasive earthworms in Brazil: diversity, distribution and effects on soils and plants. Caribbean Journal of Science. 42: 111-117.

Callaham Jr., M. A., P. F. Hendrix \& J. P. Ross. 2003. Occurrence of an exotic earthworm (Amynthas agrestis) in undisturbed soils of the southern Appalachian Mountains, USA. Pedobiologia. 47: 466-470. 
Fragoso, C., G. G. Brown, J. C. Patrón, E. Blanchart, P. Lavelle, B. Pashanasi, B. K. Senapati \& T. Kumar. 1997. Agricultural intensification, soil biodiversity and agroecosystem function in the tropics: The role of earthworms. Applied Soil Ecology. 6: 17-35.

Hendrix, P. F. 2002. Soil fauna. Pp. 45-94. In: M. E. Sumner (Ed.). Handbook of soil science. CRC Press, Boca Raton.

Hendrix, P. F. (Ed.) 2006. Biological invasions belowground: Earthworms as invasive species. Springer, Dordrecht.

Langenbach, T., M. V. S Inácio, A. M. Aquino \& B. Brunninger. 2002. Influência da minhoca Pontoscolex corethrurus na distribuição do acaricida dicofol em um Argiossolo. Pesquisa Agropecuária Brasileira. 37: 1663-1668.

Lewinsohn, T. M., A. Freitas \& P. I. Prado. 2005. Conservação de invertebrados terrestres e seus habitats no Brasil. Megadiversidade. 1: 62-69.

MMA. 2000. Avaliação e ações prioritárias para a conservação da biodiversidade da Mata Atlântica e Campos Sulinos. MMA/SBF, Brasília.

Muys, B. \& P. H. Granval. 1997. Earthworms as bio-indicators of forest site quality. Soil Biology \& Biochemistry. 29: 323-328.

Nunes, D. H., A. Pasini, N. P. Benito \& G. G. Brown. 2007. Minhocas como bioindicadoras da qualidade ambiental. Um estudo de caso na região de Jaguapitã, PR, Brasil. Pp. 467-480. In: G. G.Brown \& C. Fragoso (Eds.). Minhocas na América Latina: biodiversidade e ecologia. Embrapa Soja, Londrina.

Paoletti, M. G. 1999. Invertebrate biodiversity as bioindicators of sustainable landscapes: Practical use of invertebrates to assess sustainable land use. Elsevier, Amsterdam.

Peixoto, R. G. T. \& A. I. Marochi. 1996. A influência da minhoca Pheretima sp. nas propriedades de um latossolo vermelho escuro álico e no desenvolvimento de culturas em sistema de plantio direto, em Arapoti-PR. Plantio Direto. 35: 23-25.

RADAMBRASIL. 1983. Projeto Radambrasil: levantamento de recursos naturais. IBGE, Rio de Janeiro.

Righi, G. 1984. Pontoscolex (Oligochaeta, Glossoscolecidae), a new evaluation. Studies on Neotropical Fauna and Environment. 19: 159-177.

Righi, G. 1999. Oligochaeta. Pp. 10-21. In: C. R. F. Brandão \& E. M. Cancello (Eds.). Biodiversidade do Estado de São Paulo, Brasil: síntese do conhecimento ao final do século XX, 5 - Invertebrados terrestres. FAPESP, São Paulo.

Reynolds, J. W. \& G. A. Jordan. 1975. A preliminary conceptual model of megadrile activity and abundance in the Haliburton Highlands. Megadrilogica. 2: 1-9.

Römbke, J., S. Jänsch \& M. Garcia. 2007. Earthworm as bioindicators (in particular for the influence of Land use). Pp. 455-466. In: G. G. Brown \& C. Fragoso (Eds.). Minhocas na América Latina: biodiversidade e ecologia. Embrapa Soja, Londrina.

Santos J. C. P., D. Baretta, A. F. Manfroi, I. Bertol, C. R. D. Maluche, O. Klauberg-Filho \& F. Crestani. 2003. Fauna edáfica como bioindicadora da qualidade do solo em diferentes sistemas de preparo e cultivo. Pp. 1-4. In: Anais da XIV Reunião Brasileira de Manejo e Conservação do Solo e da Água. SBCS/UFMT, Cuiabá. CD-ROM.

Tabarelli, M. \& W. Mantovani. 1999. A regeneração de uma floresta tropical de montanha após corte e queimada (São Paulo-Brasil). Revista Brasileira de Biologia. 59: 239-250.

Veloso, H. P., A. L. R. Rangel-Filho \& J. C. A. Lima. 1991. Classificação da vegetação brasileira adaptada a um sistema universal. IBGE, Rio de Janeiro. 